\title{
Differential impairments of rat serial pattern learning and retention induced by MK-801, an NMDA receptor antagonist
}

\author{
STEPHEN B. FOUNTAIN and JAMES D. ROWAN \\ Kent State University, Kent, Ohio
}

\begin{abstract}
Three experiments investigated the role of NMDA receptor dependent systems in sequential learning and memory. Rats tracked serial patterns after systemic administration of MK-801, an NMDA receptor antagonist that blocks plasticity in the hippocampus and other structures. Experiments 1 and 2 sought to describe the effects of MK-801 on acquisition and retention of serial patterns. Patterns were 24 elements long and highly organized, with some groups receiving a "violation" element that did not fit the organization of the rest of the pattern. Experiment 3 evaluated MK-801's effects on the integration of new information that was either consistent or inconsistent with a previously learned pattern structure. The results of these experiments indicated that MK-801 interfered with serial pattern learning and, to a lesser degree, retention. Learning about structure was relatively spared, whereas learning about interruptions or violations of pattern structure was impaired. The latter differential effects are consistent with the contention in earlier literature that serial pattern learning is subserved by at least two learning/memory systems.
\end{abstract}

Many animals are able to generate elaborate sequences of behavior and can use sequential information about the order of past events to anticipate future events. How does the central nervous system organize and represent sequential information? Initial evidence supports the idea that damage involving the hippocampal system can produce selective deficits in sequential learning and memory (Cohen, 1984; Compton, 1993; Fountain, Schenk, \& Annau, 1985; Kesner \& Novak, 1982; Knopman \& Nissen, 1987; Olton, Shapiro, \& Hulse, 1984). The extent and nature of the observed deficits may depend on the formal structural properties of the sequence to be learned (e.g., Fountain, 1986). When sequences are composed of unrelated and unorganized items, the cognitive and neural processes recruited during learning may be different than when sequences are built of items related in some meaningful way and presented in a structured, organized fashion - that is, in "serial patterns." The experiments to be described begin to examine potential neural correlates of serial pattern learning as a function of pattern structure.

The idea that animals are sensitive to the structure of sequential patterns is well supported. When animals learn

This work was supported in part by the National Institute of Mental Health Grant MH48402 to S. B. Fountain. J. D. Rowan reported this work in another form as a dissertation in partial fulfillment of the doctor of philosophy degree in the Department of Psychology, Kent State University. The authors thank Peggy Ruyak and Heidi M. Carman for assistance in conducting surgery and collecting data and Don Olson for assistance in building the apparatus. Correspondence should be addressed to S. B. Fountain, Department of Psychology, Kent State University, Kent, OH 44242-0001 (e-mail: sfountai@kent.edu), or J. D. Rowan, Department of Psychology, Wesleyan College, 4760 Forsyth Rd., Macon, GA 31210-4462 (e-mail: jrowan@wesleyancollege.edu). to anticipate sequential patterns of different food quantities (Fountain, Evensen, \& Hulse, 1983; Fountain \& Hulse, 1981; Hulse \& Dorsky, 1977, 1979; Phelps \& Roberts, 1991 ) or different responses in a horizontal array (Fountain \& Rowan, 1995a, 1995b) across trials, they show convincing evidence of being sensitive to the formal structure of the patterns. The formal structure of a serial pattern is the systematic relationship or set of relationships-that is, "rules"-relating pattern elements. In particular, patterns are often composed of sets of related elements called chunks. The rules relating individual pattern elements within chunks are called lower order rules, and the rules that relate chunks or sets of chunks are called higher order rules. Recent work has shown that rats are sensitive both to lower and higher order pattern structure (Fountain \& Rowan, 1995a) and to manipulations that affect the chunking process (Capaldi, Verry, Nawrocki, \& Miller, 1984; Fountain, Henne, \& Hulse, 1984; Fountain \& Rowan, 1995a; Phelps \& Roberts, 1991; Stempowski, Carman, \& Fountain, 1999; Terrace, 1987, 1991).

Evidence indicates that more than one memory system is involved in serial pattern learning, at least one of which depends on the hippocampus (Fountain, 1986; Fountain et al., 1985; Olton et al., 1984). For example, one experiment showed that hippocampal lesions produce results predicted by the rule-learning view of serial pattern learning if one views rule induction as a process potentially dissociable from discrimination learning processes involved in serial pattern learning. Fountain et al. (1985) trained rats with long monotonically (rule-based) and nonmonotonically (unstructured) decreasing patterns created from quantities of brain-stimulation reward. Half the rats were exposed to trimethyltin (TMT), a neurotoxic 
organometal that produces damage in the limbic system, primarily in the hippocampus. TMT-exposed rats learned the rule-based monotonic pattern as fast as control rats but learned the unstructured nonmonotonic pattern slower than controls. The results fit with the notion that rule-induction processes used for learning the structure of the monotonic pattern were spared following TMT damage, whereas discrimination learning processes used in learning the unstructured nonmonotonic pattern were impaired by TMT damage. These and other data suggested that item association formation is a hippocampal-dependent process, whereas rule induction is not.

To further examine the nature of potential hippocampal involvement in serial pattern learning, in the experiments reported below, rats were administered a drug during either serial pattern acquisition or tests of serial pattern retention. The drug, MK-801 [(+)-10,11-dihydro5-methyl-5H-dibenzo(a-d)cycloheptand-5,10imine], is a noncompetitive $N$-methyl-D-aspartate (NMDA) receptor antagonist that blocks NMDA-mediated long-term potentiation (LTP) that occurs in hippocampus and other structures. NMDA-mediated LTP is a candidate neurobiological mechanism for the storage of certain types of memory by the hippocampus (Hargreaves, Cote, \& Shapiro, 1997; Teyler \& DiScenna, 1987) and other structures, such as the amygdala (e.g., Lee \& Kim, 1998; Stevens, Shapiro, \& White, 1997). In the serial pattern learning procedure (Fountain \& Rowan, 1995a, 1995b), rats were tested in an octagonal Plexiglas operant chamber equipped with a retractable lever on each wall. Each trial began with the presentation of all eight levers. The task for the rats was to learn to press the eight levers in a particular sequential pattern. Experiments 1 and 2 sought to describe the effects of MK-801 on acquisition and retention of serial patterns. Experiment 3 evaluated MK-801's effects on the integration of new information that was either consistent or inconsistent with a previously learned pattern structure.

\section{EXPERIMENT 1}

Earlier work has suggested that an intact hippocampus may be necessary for the formation of associations between events in serial patterns (Olton et al., 1984), whereas the hippocampus may not be important for rule learning in highly structured serial patterns (Fountain et al., 1985). In Experiment 1, rats were trained on two patterns: one that was structurally "perfect," and a second virtually identical to the first but containing a single element that violated the otherwise simple structure. The perfect and violation patterns were:

$$
\text { Perfect: } \quad 123234345456567678781812
$$

$$
\text { Violation: } 12323434545656767878181 \underline{8} \text {, }
$$

where the digits indicate the clockwise position of the reinforced lever for successive trials. The last " 8 " item of the violation pattern (underlined) was the violation element. Rats from one group for each pattern condition were injected with MK-80I daily before training. The rationale was that since the hippocampus has been shown to be an important structure for associative learning and memory in serial learning, then blocking the proposed mechanism of associative learning (viz., LTP) in this structure should produce an inability to learn elements or portions of patterns requiring this strategy. However, if MK-801 has little impact on rule-learning processes, then MK-801 should have little effect on learning to respond to rule-based items within chunks of the pattern.

\section{Method}

Subjects. The subjects were 24 naive male hooded rats (Rattus norvegicus) bred in-house. The rats weighed approximately $300 \mathrm{~g}$ at the time of surgical implantation of electrodes and were 80-100 days old. The rats were housed in individual wire mesh cages and were provided free access to food and water in their home cages throughout the experiment.

Apparatus. Two smaller shaping chambers $(30 \times 30 \times 30 \mathrm{~cm})$, each equipped with a single response lever and a commutating device centrally located in the ceiling, were used for shaping the leverpress response for brain stimulation reward (BSR). Each was constructed of clear Plexiglas with a floor of stainless steel rods and enclosed in a sound-attenuating shell made of particle board. The shaping chambers were housed in a room different from that of the test chamber.

The test chamber used was octagonal in shape (walls were $15 \mathrm{~cm}$ wide $\times 30 \mathrm{~cm}$ tall; the chamber measured approximately $40 \mathrm{~cm}$ between parallel walls) and was composed of clear Plexiglas walls and a floor of hardware cloth. A retractable response lever was centered on each wall $5.0 \mathrm{~cm}$ above the floor. Each lever required approximately $0.15 \mathrm{~N}$ of force for activation. The rats were connected to a stimulator by way of a flexible cord and a commutating device centered in the ceiling of the chamber. The operant chamber was located in a testing room approximately $2 \times 2.6 \mathrm{~m}$.

Procedure. Each rat was anesthetized by intraperitoneal (i.p.) injection of $36.5 \mathrm{mg} / \mathrm{kg}$ Ketamine and $3.65 \mathrm{mg} / \mathrm{kg}$ Xylazine injected in a constant volume of $2 \mathrm{ml} / \mathrm{kg}$ bacteriostatic water. A twisted stainless steel bipolar electrode (Plastic Products MS303/1) was then implanted unilaterally in the posterior lateral hypothalamus of each rat. With the skull level, the coordinates were $4.5 \mathrm{~mm}$ posterior to the bregma, $1.5 \mathrm{~mm}$ lateral to the midline, and $8.5 \mathrm{~mm}$ below the surface of the skull.

Within 2 weeks following implantation of electrodes, the rats were placed in the shaping chamber and were shaped to press a response lever for BSR. A $60-\mathrm{Hz}$ sinusoidal pulse train of $250-\mathrm{msec}$ duration from a constant current source of $30-100 \mu \mathrm{A}$ was delivered for shaping and for each leverpress. The rats that failed to leverpress more than 1,000 times in one of two 30 -min sessions were excluded from the experiment.

Throughout the experiment, the pattern training procedure remained constant. At the beginning of each trial, all eight levers were inserted into the chamber. When the rat produced a response on the correct lever, all levers were retracted from the chamber and a pulse of BSR was administered. When the rat produced an incorrect response, all levers except the correct one were retracted and no reward was administered until the rat pressed the correct lever. This correction procedure ensured that the rats received feedback regarding the correct lever on each trial. An intertrial interval (ITI) of 1 sec was imposed except where "phrasing" conditions called for longer intervals. The rats typically required shaping in this procedure for 1-2 days. Those that failed to acquire the task in 2 days were excluded from the experiment.

The rats performed 50 repetitions of their patterns each day. The patterns that the rats were required to produce were: 


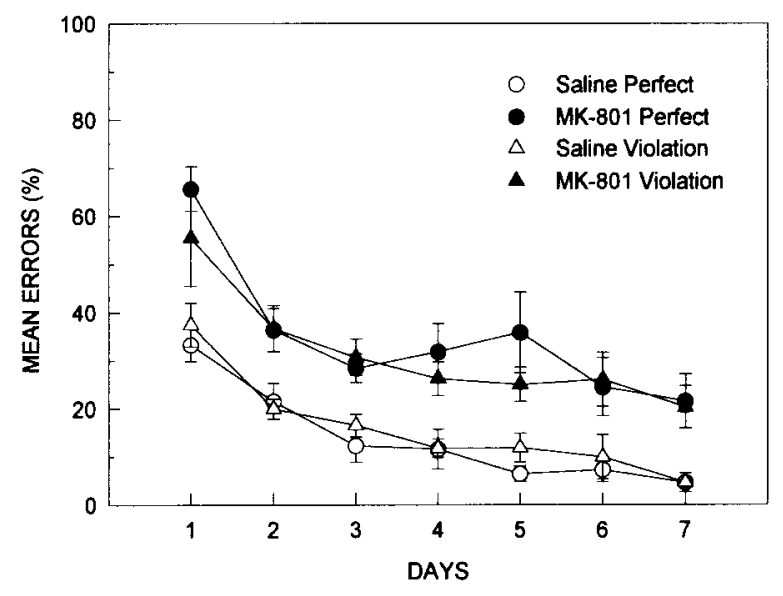

Figure 1. Acquisition for the saline and MK-801 groups for perfect and violation patterns over the 7 days of Experiment 1. Percentages of daily mean errors were averaged across items of the patterns.

Perfect runs: $\quad 123234345456567678781812$

Violation runs: $12323434545656767878181 \underline{8}$,

where the integers refer to the clockwise positions of the eight levers and the spaces indicate $3-\mathrm{sec}$ pauses that separate three-element chunks. Note that the last element of the violation pattern is inconsistent with the structure of the rest of the pattern. Injections of either MK-801 $(0.0625 \mathrm{mg} / \mathrm{kg}$ body weight in a solution of $0.0625 \mathrm{MK}$ $801 \mathrm{mg} / \mathrm{ml}$ bacteriostatic $\mathrm{H}_{2} \mathrm{O}$ ) or an equivalent volume of saline were made i.p. 30 min prior to training on each of the 7 days of the experiment. Earlier work has shown that MK-801 at this dose continues to produce profound learning deficits in rats even after more than 2 months of daily administration (Shapiro \& Caramanos, 1990), indicating that tolerance should not be a factor in this experiment.

\section{Results}

In all reported analyses, main effects and interactions were considered significant if $p<.05$. An analysis of variance (ANOVA) was conducted on the rats' mean error rates for the entire pattern collapsed across all elements of the pattern for Days 1-7 of testing (Figure 1). The ANOVA revealed a significant main effect for drug $[F(1,20)=$ $25.79]$, indicating that MK-801 increased errors for both the perfect pattern and the violation pattern across the 7 days of the experiment. No other significant main effects or interactions were observed $(p>.05)$.

An additional ANOVA was conducted on the rats' mean error rates for the last element of each pattern for Days 1-7 of Experiment 1 (Figure 2) in order to evaluate acquisition of the terminal element of each pattern under a drug or nondrug state. It revealed main effects for pattern $[F(1,20)=279.01]$ and $\operatorname{drug}[F(1,20)=25.77]$. A significant interaction for pattern $\times$ drug $\times$ days $[F(6,120)=$ 15.61] was also indicated. Planned comparisons to evaluate the error rates on a day-by-day basis were computed on the basis of the appropriate error terms from the foregoing ANOVA using Fisher's least significant difference (LSD) procedure (Kirk, 1968). Results from the planned comparisons of the pattern $\times$ drug $\times$ days factor for the last element of the pattern revealed that, for Days 1,2 , and 5 , the MK-801 perfect group made significantly more errors than the saline perfect group (Figure 2, top panel). However, on Days 1, 4, 5, 6, and 7, the MK-801 violation group made significantly more errors than the saline violation group, and the differences between the two groups increased during the course of the experiment (Figure 2, bottom panel). In fact, on Day 7 (the last day of the experiment), the MK-801 violation group made four times as many errors as the saline violation group, and the MK-801 violation group was still performing worse than chance $(89.7 \%)$ on Day 7 . The saline violation group, however, showed significant improvement on the last element beginning on Day 4 and was performing at $22 \%$ errors for this element on Day 7. In addition, planned comparisons on daily means within each of the groups revealed that only the MK-801 violation group failed to show any significant improvement over the 7 days of the experiment.

Figure 3 shows the rats' mean error rates for each element of the 24-element pattern for Day 7. An ANOVA conducted on the rats' error rates for each element of the 24-element pattern for Day 7 revealed significant main effects for drug $[F(1,20)=18.92]$, chunk $[F(7,140)=$ $10.23]$, and element of chunks $[F(2,40)=25.25]$. Interactions were found for pattern $\times$ chunk $[F(7,140)=$ $11.74]$, drug $\times$ chunk $[F(7,140)=5.76]$, drug $\times$ element $[F(2,40)=7.31]$, chunk $\times$ trial $[F(14,280)=11.09]$, pattern $\times$ drug $\times$ chunk $[F(7,140)=9.85]$, pattern $\times$ chunk $\times$ element $[F(14,280)=10.27]$, drug $\times$ chunk $\times$ trail $[F(14,280)=6.72]$, and pattern $\times$ drug $\times$ chunk $\times$ element $[F(14,280)=5.70]$. All other main effects and interactions were not significant $(p>.05)$. Planned comparisons to evaluate the error rates on an element-byelement basis were computed on the basis of the appropriate error terms from the foregoing ANOVA using the Fisher's LSD procedure.

The rats in the MK-801 perfect group were not significantly impaired on the terminal element ("2") on Day 7 , relative to the rats in the saline perfect group. However, the MK-801 violation group was significantly impaired on the last element (" 8 "), relative to the saline violation group. The rats in both MK- 801 groups also showed impairments in the ability to anticipate Element 1 of each three-element chunk, which appears as elevated errors for MK-801 groups on the first element after the break in each line in Figure 3.

The rats' response latency data were also analyzed. An ANOVA was calculated for the rats' response latency data on an element-by-element basis for Day 7. The ANOVA indicated significant main effects for pattern $[F(7,140)=$ $2.04]$ and element $[F(2,40)=6.72]$. Significant interactions were found for drug $\times$ chunk $[F(7,140)=3.13]$ and drug $\times$ chunk $\times$ element $[F(14,280)=1.93]$. No other main effects or interactions were significant. Planned comparisons revealed that, on Element 1 of Chunk 1 of both the perfect pattern and the violation pattern, the MK801 rats responded significantly faster than the saline rats. 

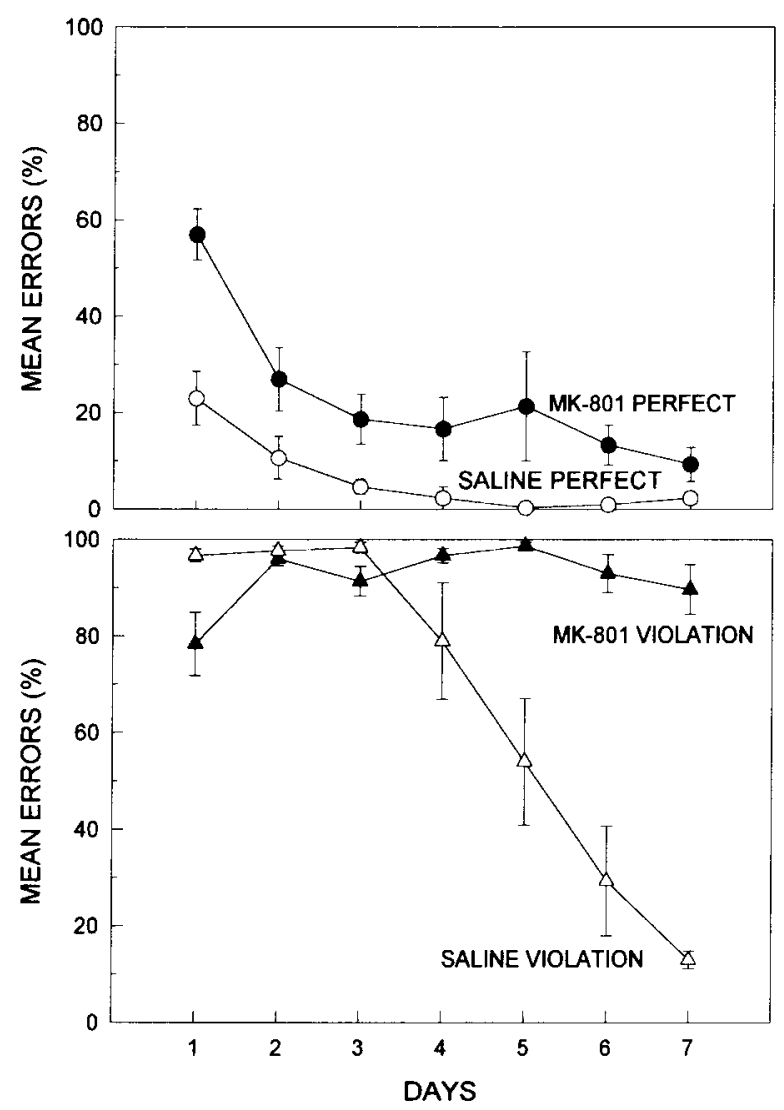

Figure 2. Acquisition of the last element of the perfect pattern (top panel) and violation pattern (bottom panel) over the 7 days of Experiment 1 for the saline and MK-801 groups. The last element was structurally consistent in the perfect pattern, and it was the violation element in the violation pattern. Daily mean errors are shown for the last element of the pattern only.

This indicated that the MK-801 significantly decreased the rats' latency to respond at the beginning of the pattern. These results did not parallel the error rate data. Under MK-801, error rates increased at the chunk boundaries and at the violation.

We also examined the rates of different types of responses that the rats made on pattern elements that were most difficult to learn - namely, Element 1 of chunks and the violation element - on the last day of acquisition (Day 7). Responses were classified as correct responses, responses that were overextensions of the lower order rule (e.g., a "4" response following the 123 series of Chunk 1), "accuracy" errors (responses one to the left and right of the correct lever), or "other" errors. All groups rarely committed overextension errors at chunk boundaries immediately following phrasing cues (less than $3 \%$ of the time in all groups). The most common errors at chunk boundaries were accuracy errors. However, for the violation element, on Day 7, the MK-801 violation group committed many more overextension errors at the violation- " 2 " after the 81 series ( $63 \%$ of responses on this element) - than did the saline violation group ( $6 \%$ of responses). Thus, MK-801 impaired learning for both chunk boundaries and the violation element, and it caused a dramatic increase in the rate of overextension errors on the violation element, relative to controls.

\section{Discussion}

The saline perfect group and the saline violation group were trained with the same patterns as and under conditions virtually identical to those of the "perfect runs" and "violation runs" groups, respectively, reported by Fountain and Rowan (1995b). The only difference in Experiment 1 was that the rats received saline injections daily $30 \mathrm{~min}$ before testing. The results for the saline perfect rats and the saline violation rats paralleled those reported by Fountain and Rowan (1995b). Specifically, the rats in both the saline perfect condition and the saline violation condition found elements within chunks easier to learn than elements immediately after chunk boundaries - that is, Elements 2 and 3 of chunks were easier than Element 1 of chunks. Finally, the violation element was the most difficult to learn, and errors on the violation trial were mostly errors of overextension of the within-chunk rule (e.g., a "2" response after the 81 series of Chunk 8).

Fountain and Rowan (1995b) interpreted this pattern of results as indicating that rats first induced a structural representation of the pattern and then later learned the "exception-to-the-rule" represented by the violation element (cf. Fountain \& Rowan, 1995a). The saline-injected rats in Experiment 1 mastered all elements of perfect and violation patterns by Day 7 , including the difficult violation element (see Figure 3). In contrast, the rats in the MK-801 groups showed deficits in acquisition on both perfect and violation patterns, but the effects of MK-801 were not evenly distributed throughout the patterns. Although there may have been some slowing of acquisition for elements within pattern chunks (Elements 2 and 3 of chunks), the MK-801 rats in both pattern conditions eventually learned within-chunk elements to well above chance levels (to approximately 10\% errors). However, severe and persistent impairments were observed for chunk boundary elements (Element 1 of three-element chunks) for both the perfect pattern and the violation pattern. In the violation pattern, performance on the violation element was also severely impaired.

Temporal phrasing cues have been shown to overshadow other cues that may control anticipation at chunk boundaries (Capaldi et al., 1984; Stempowski et al., 1999). Earlier studies have shown that the temporal phrasing cues used in Experiment 1 practically eliminate overextension errors that would otherwise have occurred at high rates on Element 1 in unphrased patterns (Stempowski et al., 1999). However, this effect is independent of the ability of temporal phrasing cues to signal the next response (cf. Fountain, Krauchunas, \& Rowan, 1999). Because the pattern of Experiment 1 was "phrased" by temporal pauses at chunk boundaries, temporal cues came to control the saline-injected rats' responses on Element 1 of chunks, 

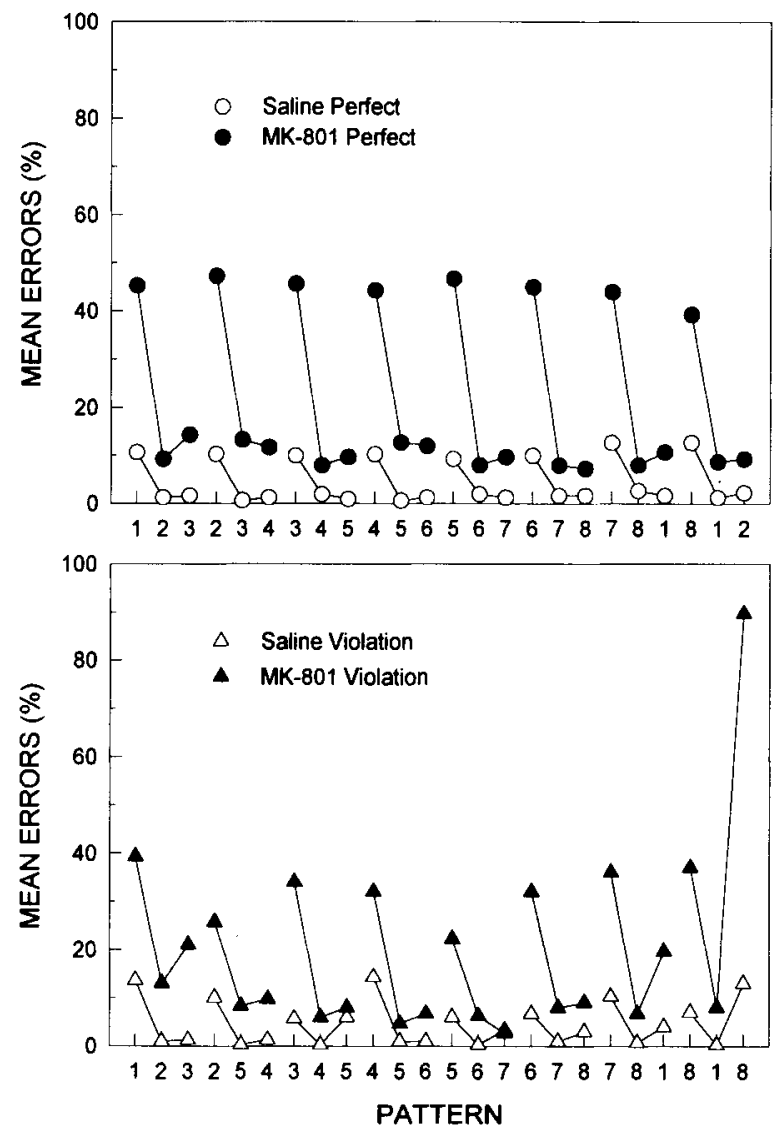

Figure 3. Mean pattern tracking errors for the perfect pattern (top panel) and violation pattern (bottom panel) for the saline and MK-801 groups in Experiment 1. Errors were averaged across pattern presentations of Day 7, the last day of the experiment.

the element immediately after the cue in each chunk. In addition, because of the presence of temporal cues, the rats produced very few overextension errors on Element 1 of three-element chunks, such as "4" after the 123 chunk. MK-801 impaired acquisition of temporal cue control over responding on Element 1 of chunks. In other words, MK-801 impaired the discrimination learning processes that mediated rats' performance at chunk boundaries. MK-801 did not effect how the presence of temporal cues reduced overextension error rates on Element 1 of chunks.

In the violation pattern, in addition to a deficit in acquisition of Element 1 of chunks, performance on the violation element was also severely impaired: The MK-801 rats never performed above chance on the violation element during the 7 days of the experiment. Performance on the violation element had to be controlled entirely by discriminative stimuli since the location of the violation element could not be predicted by pattern structure alone. In this task, cues supporting anticipation of the violation element could have included combinations of sequential elements (at least three would be required), temporal or sequential position cues, or "lever" cues that might have been proximal (apparatus) or distal (spatial) cues. MK-801 clearly interfered with the discrimination learning processes that mediated the saline control rats' ability to produce the correct response on precisely the correct trialthat is, on the violation trial.

Perhaps the most interesting finding in Experiment 1 was that the MK-801 violation rats persisted in producing high rates of the overextension error on the terminal violation trial, with no signs of learning. That is, instead of learning to produce the required " 8 " response after the 81 sequence of the violation chunk, they persistently produced a " 2 " response. As indicated earlier, the " 2 " response was structurally consistent with responses that the rats were required to learn in all other chunks of the pattern. It should be noted that, in the perfect pattern, where the terminal element was the structurally consistent " 2 " response, the MK-801 rats performed as well as the saline control animals by Day 3 of the experiment. This finding supports the idea that MK-801 had somewhat selective effects on the learning processes used to encode the structurally inconsistent violation element of the violation pattern. The fact that rule-consistent overextension errors predominated on the violation element is strong evidence that rule learning processes were spared by MK801 at the same time that discrimination learning processes were impaired.

The focus on discrimination learning processes as the target of MK-801 effects may raise the question of why Element 1 of chunks (chunk boundaries) in Experiment 1 were not learned more rapidly and were not better anticipated by the MK-801 rats. Chunk boundaries are not predicted by lower order structure (the " +1 " rule of withinchunk elements), but they can be encoded as higher order transitions in pattern structure that, in Experiment 1, were quite well organized and repetitive throughout the pattern. According to the view that rule induction processes were spared by MK-801, learning should have been spared for higher order structural features, such as chunk boundaries. It should be noted that learning was in fact observed for chunk boundaries: The rats in both MK-801 pattern groups improved from approximately $70 \%$ errors to approximately $40 \%$ errors on Element 1 of chunks over the course of the experiment. However, learning was poorer in the MK-801 groups relative to the corresponding saline controls, presumably because the MK-801 rats did not have the advantages afforded by being able to use phrasing cues (Fountain et al., 1984; Stempowski et al., 1999).

The results of Experiment 1 are consistent with a dualprocess perspective that posits the existence of independent discrimination learning and rule learning processes in rat serial pattern learning, as proposed earlier (Fountain, 1986, 1990; Fountain \& Rowan, 1995a; Fountain et al., 1985). From the dual-process perspective, when MK- 801 blocked cue-item association formation, the rats could not acquire responses that depended on specific temporal, spatial, or other cues in the environment. Such responses were required at chunk boundaries and on the 

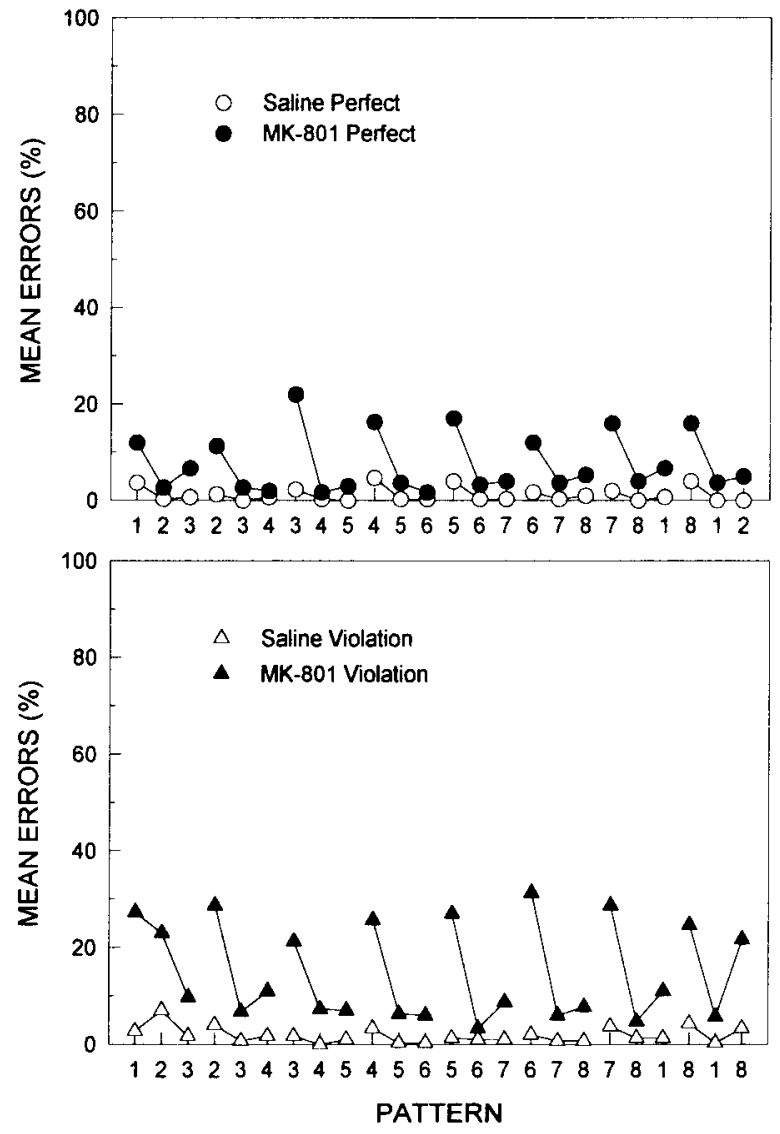

Figure 4. Mean pattern tracking errors for the perfect pattern (top panel) and violation pattern (bottom panel) for the saline and MK-801 groups in Experiment 2. Errors were averaged across the 50 patterns of the injection day of the experiment.

violation element, both locations in the pattern where lower order pattern structure failed to direct the proper response. However, according to this view, learning for elements within chunks was relatively unimpaired because MK-801 spared rule induction processes.

\section{EXPERIMENT 2}

Another prediction that follows from the hypothesis that MK-801 impairs the associative processes mediating discrimination learning is that MK- 801 should have effects only during the acquisition of the task. MK-801, as mentioned earlier, impairs induction of LTP in the hippocampus by blocking activity-dependent NMDA receptors. Once a rat has learned a serial pattern, for example, the synaptic changes that are thought to be the basis of NMDA-mediated learning will already have taken place, and MK-801 should have minimal impact on performance of already-learned behavior. Thus, if the deficits observed in acquisition of chunk boundaries and the violation element in Experiment 1 were due to the drug's effects on hippocampal LTP induction, then MK-801 should have little or no effect on performance of an already-learned pattern. This idea was tested in Experiment 2, in which rats were trained to a strict criterion on the perfect and violation patterns of Experiment 1 and were then tested on performance of the training patterns under MK-801.

\section{Method}

Subjects. The subjects were 24 naive male hooded rats (6 were randomly assigned to each of the four experimental conditions). The rats were prepared and maintained as in Experiment 1.

Apparatus. The apparatus was the same as that used in Experiment 1.

Procedure. All procedures for electrode implantation and leverpress shaping prior to the experiment proper were identical to those used in Experiment 1. The rats were trained to a criterion of no more than $10 \%$ errors on any single element of either the perfect pattern or the violation pattern of Experiment 1. On the next day after meeting criterion, half the rats received an i.p. injection of $0.0625 \mathrm{mg} / \mathrm{kg}$ body weight MK-801 (the same dose used in Experiment 1) or a saline control injection 30 min prior to testing on the training pattern. Each rat performed 50 repetitions of its pattern each day of the training and transfer phases.

\section{Results}

The rats in the perfect group took an average of 7.74 days to reach the criterion of no more than $10 \%$ errors on any single element of the pattern. The rats in the violation group reached the criterion in an average 12.09 days. On the day after reaching criterion, the rats were injected with MK-80l and were tested using the same procedures 30 min later. On the injection day, the rats produced the data shown in Figure 4. The ANOVA performed on the element-by-element error rate data revealed significant main effects for drug $[F(1,20)=4.61]$, chunk $[F(7,140)=$ $3.57]$, and element $[F(2,40)=11.39, p<.05]$. Interactions were found for pattern $\times$ chunk $[F(7,140)=4.49]$, drug $\times$ element $[F(2,40)=6.07]$, chunk $\times$ element $[F(14,280)=$ 1.99], and pattern $\times$ drug $\times$ element $[F(14,280)=3.07]$. All other main effects and interactions did not prove to be significant $(p>.05)$. Planned comparisons revealed differential effects of MK- 801 on the three elements of chunks: MK-801 significantly increased errors on Elements 1 and 3 of chunks in the violation pattern and on Element 1 of the perfect pattern relative to corresponding elements of the violation and perfect patterns, respectively, for the rats in the saline groups.

The ANOVA performed on the latency data revealed only one significant interaction: the chunk $\times$ element interaction $[F(14,280)=3.44]$. The fact that there were no significant main effects or interactions involving drug as a factor indicated that MK- 801 did not produce an effect on the rats' ability to simply perform the leverpress response.

The rats' responses for the most difficult elements of the pattern were examined (viz., at chunk boundariesElement 1 of chunks - and the violation element). Responses were classified into four categories as in Experiment 1: correct responses, overextension errors, accuracy errors, and "other" errors. As in Experiment 1, the most common errors at chunk boundaries were accuracy errors 
(responses one to the left and right of the correct lever), and overextension errors were rare (less than $1 \%$ of responses) even under MK-801. In contrast to Experiment 1 , the most common errors on the violation element were not overextension errors. Instead, in Experiment 2, the most common errors on the violation element, as at chunk boundaries, were accuracy errors, and overextension errors were rare even under MK-801, accounting for only $4 \%$ of responses.

\section{Discussion}

In Experiment 2, MK-801 produced small but reliable effects on the rats' retention performance of serial patterns. For the MK-801 perfect group, the drug selectively disrupted performance on the first element of chunks (i.e., at chunk boundaries). However, performance on the second and third elements of chunks was not affected. Performance of the MK-801 violation group was similar to that of the MK-801 perfect group at chunk boundaries, but MK-801 also increased errors on Element 3 of threeelement chunks. The response latency data indicated that even though the rats' performance on the pattern was not as accurate for specific elements, the MK-801 rats did not take significantly more or less time to make responses relative to the saline controls.

Examination of the types of responses that the rats made during testing revealed results similar to those found in Experiment 1. MK-801 did not affect the number of overextension errors observed at the chunk boundaries. However, in Experiment 2, MK-801 also did not increase the number of overextension errors for the violation element, as it did in acquisition in Experiment 1. Thus, examination of the rats' responses at the chunk boundaries adds some support to the hypothesis that MK-801 may not affect rats' retention of the pattern but instead affects their accuracy at chunk boundaries and the violation element.

Even though performance deficits were observed, it should be noted that, even in the worst cases, the rats under MK-801 performed well above chance throughout their patterns. The rats' errors under MK-801 can generally be described as accuracy errors, and these errors simply occurred at a higher rate in MK-801 conditions relative to saline conditions. That is, MK-801 appeared to produce a small quantitative effect on performance rather than qualitative changes in the kind or distribution of errors that the rats produced. The results suggest that MK-801 affected pattern tracking performance rather than the rats' access to the cognitive structures mediating tracking of the welllearned patterns at the end of training.

\section{EXPERIMENT 3}

Experiment 3 examined the role of NMDA receptor mediated plasticity when new serial pattern information is added to old. Rats were first trained to a high criterion on a pattern consisting of the first seven chunks of the perfect pattern used in Experiments 1 and 2: 123234345 456567678781 . After rats learned the pattern, they were transferred to one of two new patterns that contained all elements of the first pattern and an additional chunk of three additional elements. The three added elements were structurally consistent with the first pattern (viz., 812), making it structurally perfect, or they contained a violation of the pattern structure learned in training (viz., 818). The resulting perfect and violation patterns in transfer were the same as those used in Experiments 1 and 2. On the day of transfer, half the rats in each transfer pattern condition were injected with MK-801, in order to determine the effects of NMDA receptor dysfunction on rats' ability to integrate structurally consistent or inconsistent new information with an already-learned pattern.

\section{Method}

Subjects. The subjects were 24 naive male hooded rats ( 6 were randomly assigned to each of the four transfer conditions). The rats were prepared and maintained as in Experiments 1 and 2.

Apparatus. The apparatus was the same as that used in Experiments 1 and 2.

Procedure. All procedures for electrode implantation and leverpress shaping prior to the experiment proper were identical to those used in Experiments 1 and 2. The rats were trained to a criterion of no more than $10 \%$ errors on any single element of the first seven chunks (i.e., the first 21 elements) of the patterns used in Experiments 1 and 2: 123234345456567678781 , where, as before, integers refer to the clockwise positions of the eight levers and spaces indicate 3-sec pauses that separate three-element chunks. However, in Experiment 3, the interval between patterns was $10 \mathrm{sec}$ rather than $3 \mathrm{sec}$. All other ITIs were $1 \mathrm{sec}$.

On the next day after meeting criterion, half the rats were transferred to the perfect pattern, and the other half were transferred to the violation pattern. That is, one three-element chunk was added to each rat's pattern, with a 3 -sec phrasing cue before the added chunk. For half the rats, the three added elements were structurally consistent with the training pattern (viz., 812), making it structurally perfect. For the other half, the added chunk contained a violation of the pattern structure learned in training (viz., 818). On the day of pattern transfer, half of each pattern group received an i.p. injection of $0.0625 \mathrm{mg} / \mathrm{kg}$ body weight MK-801 (the same dose used in Experiments 1 and 2) or a saline control injection 30 min prior to testing on the training pattern. Each rat performed 50 repetitions of its pattern each day of the training and transfer phases.

\section{Results}

The rats in Experiment 3 reached criterion of no more than $10 \%$ errors on any element of the training pattern in an average of 11.84 days. The data obtained on the pattern transfer and injection day of the experiment were analyzed to assess MK-801 effects on element-by-element error rates or effects on the rats' latency to respond on an element-by-element basis.

The ANOVA performed on the element-by-element error rate data for the transfer day (see Figure 5) showed significant main effects for pattern $[F(1,20)=6.41]$, drug $[F(1,20)=12.97]$, chunk $[F(7,140)=39.78]$, and element $[F(2,40)=11.22]$. The ANOVA demonstrated significant interactions for pattern $\times$ chunk $[F(7,140)=24.65]$, chunk $\times$ element $[F(14,280)=12.79]$, pattern $\times$ drug $\times$ element $[F(2,40)=3.22]$, and pattern $\times$ chunk $\times$ element $[F(14,280)=17.43]$. All other main effects and interactions were not significant $(p>.05)$. Planned compar- 

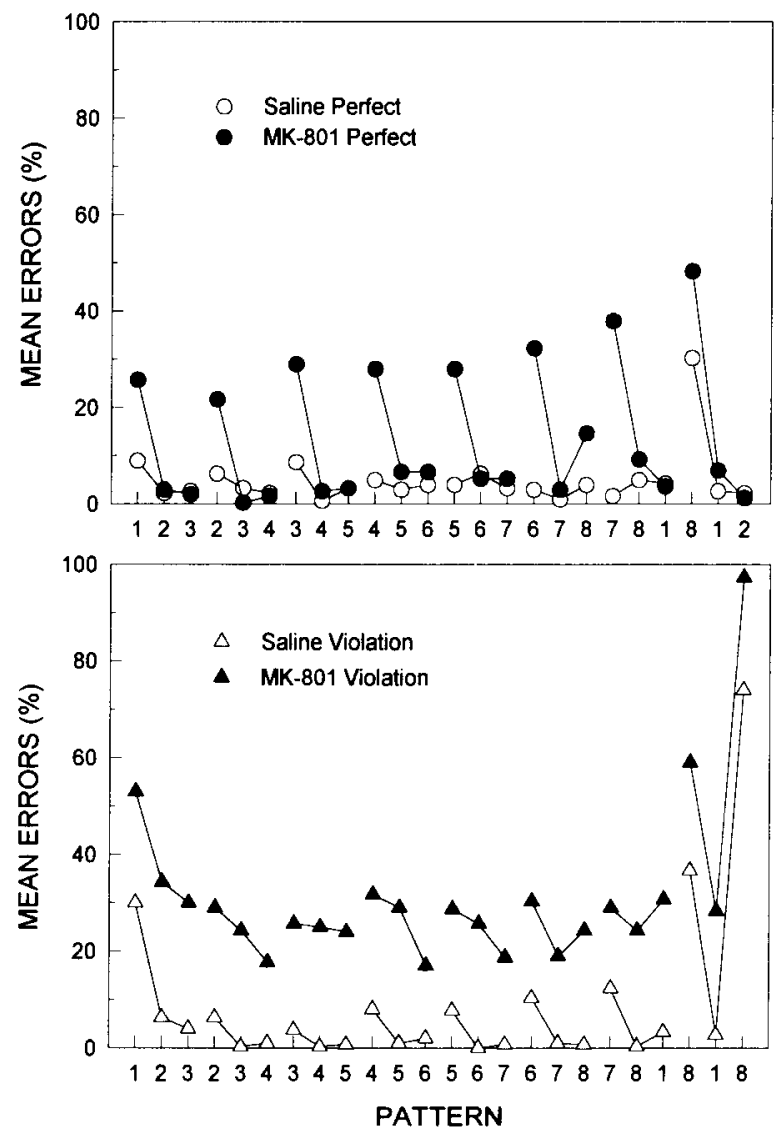

Figure 5. Mean pattern tracking errors for saline and MK-801 perfect groups (top panel) and saline and MK-801 violation groups (bottom panel) for the transfer and injection day of Experiment 3. Errors were averaged across the 50 patterns of the transfer and injection days.

isons indicated differential effects of MK-801 for perfect and violation patterns. For the perfect pattern, MK-801 significantly increased errors on Element 1 of all chunks relative to saline controls, but not on Elements 2 and 3 of three-element chunks. It is interesting to note that this pattern of effects was true both for old chunks and for the new structurally consistent chunk added on the day of injection. For the violation pattern, MK-801 significantly increased errors on all three elements of all chunks relative to saline controls. That is, MK- 801 produced a consistent deficit on every element of the violation pattern for both the old chunks and the newly added violation chunk of the pattern. The violation element was the site of high error rates as expected in the saline condition, but MK-801 produced approximately the same deficit on the violation element as it did elsewhere throughout the pattern. Comparisons between perfect and violation MK-801 groups showed that MK-801 increased errors on Elements 2 and 3 of chunks to a greater degree in the violation pattern group than in the perfect pattern group, but performance on Element 1 was not significantly different between pattern groups.

Analysis of response latency data on an element-byelement basis showed main effects for chunk $[F(7,140)=$ $2.34]$ and element $[F(2,40)=14.13]$. All other main effects and interactions were not significant, indicating that the drug did not produce a consistent effect on the rats' latency to respond.

The rats' responses for the most difficult elements of the pattern were examined (viz., at chunk boundariesElement 1 of chunks-and the violation element). Responses were classified into four categories, as in Experiments 1 and 2: correct responses, overextension errors, accuracy errors, and "other" errors. As in Experiments 1 and 2 , the most common errors at chunk boundaries, even for the added chunks in both the perfect pattern and the violation pattern, were accuracy errors (responses one to the left and right of the correct lever). Overextension errors were rare (less than $1 \%$ of responses) for both pattern conditions even under MK-801. In contrast to Experiment 2 , however, the most common errors on the violation element in both MK-801 and saline conditions were overextension errors. In this regard, MK- 801 produced effects on the added chunk that paralleled its effects on acquisition in Experiment 1.

\section{Discussion}

In Experiment 3, the rats were challenged to incorporate a new pattern chunk into a well-learned pattern at the same time that they experienced an MK-801 drug challenge. In the perfect group, when the added chunk of information was structurally consistent with the welllearned pattern, the effects of MK-801 were very similar to the effects of the drug on retention in Experiment 2. MK-801 produced a selective decrease in the rats' accuracy on Element 1 of each three-element chunk but produced virtually no change in accuracy on the remaining two elements. As in Experiment 2, the drug did not produce a consistent effect on the rats' latency to respond, which indicates that the rats' deficits in performance cannot easily be attributed to changes in the their physical ability to respond (e.g., due to fatigue, hypoactivity, hyperactivity, changes in coordination, etc.).

The most interesting result was observed in the groups that received an added violation chunk in transfer. The saline violation group had difficulty learning the new information. Error rates were high on the first element of the new chunk, as in the perfect group, and on the violation element on the last element of the new chunk. However, errors were low in the rest of the pattern. The effect of adding a violation chunk under saline was essentially limited to the elements of the newly added chunk. In contrast, the MK-801 violation group showed not only a large disruption for elements in the new chunk but also a deficit in performance for every element of the portion of the pattern that was well learned before transfer. The latter 
effect was not observed when a perfect chunk was added, which had the first two elements in common with the violation chunk; hence, this effect can be accounted for only by the addition of the violation element. This effect was not observed in Experiments 1 and 2.

The results of Experiment 3 suggest that MK-801 impaired a memory system that normally mediates integration of structurally inconsistent information with rulebased representations of serial patterns. The saline violation group did not show any deficits on the performance of the old material, whereas the MK-801 violation group did. The memory system remaining under MK-801 appears able to accommodate new structural information if it is consistent with the existing representation of the sequence, but not if the new information is inconsistent with that representation. In fact, Experiment 3 showed that structurally inconsistent information can disrupt performance based on earlier learning when NMDA-mediated learning is impaired. This suggests that, in normally functioning rats, two behavioral systems interact so that integration of new inconsistent information does not disrupt the representation of old information and that NMDAmediated plasticity plays an important role in this process.

\section{GENERAL DISCUSSION}

In three experiments, MK-801 was shown to have significant effects on acquisition of serial pattern information but little effect on serial pattern retention. Furthermore, MK-801 disrupted acquisition of the sort characterized as associative discrimination learning - that is, learning to use stimuli to cue specific responses at chunk boundaries and at the violation element. In contrast, MK-801 did not disrupt acquisition of the sort characterized as rule learning (cf. Fountain et al., 1985) - that is, learning about the consistent " +1 " or "right-turn" rule that characterized elements within chunks. In Experiment 1, the rats were trained on two patterns: one that was structurally perfect, and a second virtually identical to the first but containing a single element at the end of the pattern that violated the otherwise simple structure. The rats from one group for each pattern condition were injected with MK801 daily before training. MK-801 had little effect on learning to respond to rule-based items within chunks. However, it did impair responding at points where lower order rules were violated (viz., on the first trial of each new chunk and, most dramatically, for the violation element). Although the rats showed no signs of learning to respond to the violation element, throughout the experiment they produced rule-based errors on the violation trial at the end of the sequence. The results are strong evidence that when NMDA receptor mediated plasticity is impaired by MK-801, perhaps in hippocampus, the discrimination learning processes necessary to track violations of pattern structure are impaired. However, MK-801 spared the rule-learning processes necessary to induce pattern structure and extrapolate the sequence on the violation trial.

Experiment 2 showed that MK-801 produced selective effects on the retention of the same patterns that were used in Experiment 1. For the perfect group, the drug selectively disrupted performance on the first, or boundary, element of each three-element chunk. However, the performance on the second and third elements of each chunk was not affected. Even though performance deficits were observed, the rats under MK-801 performed well above chance throughout their patterns. The results indicate that MK-801 affected pattern tracking performancespecifically, the rats' ability to direct responses accurately, not the cognitive structures used to track their patterns.

Experiment 3 examined the role of NMDA receptor mediated plasticity when new serial pattern information is added to old. The rats were first trained to a high criterion on a pattern consisting of the first seven chunks of the perfect pattern used in Experiments 1 and 2 and then were transferred to one of two new patterns that contained all elements of the first pattern and a new eighth chunk of three additional elements. The three added elements either were structurally consistent with the first pattern, making it structurally perfect, or contained a violation of the pattern structure learned earlier. On the day of transfer, half the rats were injected with MK-801. When a structurally consistent chunk was added, the effects of MK-801 were very similar to the effects of the drug on retention in Experiment 2. That is, MK-801 produced a selective decrease in the rats' accuracy on the first elements of each chunk but produced virtually no change in performance on the remaining two elements of the three-element chunks. The most interesting result occurred when a structurally inconsistent chunk was added in transfer. Although saline controls showed difficulty in learning the new violation chunk, there was little effect on the rest of the pattern. However, MK-801 dramatically disrupted performance for elements both in the new violation chunk and throughout the rest of the pattern. When this effect is compared with the effects of MK- 801 in the perfect chunk transfer, the effect can be accounted for only by the addition of the terminal violation element.

\section{MK-801 and Possible \\ Performance (Nonmnemonic) Effects}

The disruption in learning and performance of these patterns cannot be easily explained by other nonspecific mechanisms, such as changes in motivation or changes in activity level, which might affect performance. It has been previously reported that MK- 801 does not decrease rats' sensitivity to BSR; in fact, it increases the rewarding quality of BSR (Carlezon \& Wise, 1993; Herberg \& Rose, 1989; Olds, 1996). Similarly, in a study using the same stimulators and other equipment used for reinforcing electrical stimulation in the experiments reported here, it was found that the dose of MK-801 used in Experiments 
1-3 above increased rats' sensitivity to BSR (Rowan, Toner, \& Fountain, 1992). Rats should then be more willing to work for BSR under the influence of MK-801 than before it is administered or under saline injections. Therefore, a decrease in motivation to accurately perform the task does not seem to be a likely interpretation of the results. Also, a change in rats' overall motivation to perform the task would not be expected to result in a specific impairment of their ability to learn or retain the patterns but should instead result in an overall general deficit in responding. Changes in rats' ability to respond, resulting from a decrease in coordination, hyperactivity, hypoactivity, and so forth, cannot be easily supported either. Finally, MK-801 did not significantly change rats' speed in responding consistently in any of the experiments, but it did impair accuracy consistently in all of the experiments, and change in activity level or a change in coordination would be expected to change the rats' response latencies. Like a change in motivation, these factors would also be expected to produce a general effect, not specific to particular pattern elements. This type of effect was not observed in these experiments.

The lack of nonspecific nonmnemonic effects on behavior for the dose of MK-801 used in Experiments 1-3 is consistent with the results of other experiments that have used the same dose and found no motor impairment (cf. Caramanos \& Shapiro, 1994). This is important because MK-801 effects on "working" memory have been observed only for relatively high doses that produce motor impairments (Caramanos \& Shapiro, 1994; Shapiro \& Caramanos, 1990; Shapiro \& O'Connor, 1992; White \& Best, 1998), none of which were expected or observed in these experiments.

\section{MK-801 Effects and Discrimination \\ Learning Views of Sequential Learning}

One of the most successful of current models of sequential learning is Capaldi's discrimination learning view (e.g., Capaldi, 1985, 1994), which proposes that pattern tracking in sequential tasks is mediated by rats' memories of past events and their capacity to signal succeeding events through interitem associations. According to this view, sequential elements and phrasing cues are best thought of as discriminative stimuli that come to signal forthcoming events. This model has been used to describe a variety of sequential behavior, including acquisition of serial patterns composed of items varying in discriminability, pattern "generalization," pattern "extrapolation," and the effects of phrasing cues, by appealing to mechanisms of discrimination learning (Capaldi \& Molina, 1979; Capaldi et al., 1984; Haggbloom, 1985; Haggbloom \& Brooks, 1985).

The idea that serial learning is mediated by discrimination learning alone cannot readily account for two effects of MK- 801 on serial pattern learning. First, the fact that MK-801 produced selective deficits in performance that were correlated with pattern structure is not easily explained by the discrimination learning view. This view posits that the sequence is learned by the formation of stimulus-response associations formed between the elements of the sequence or between extrasequence cues, such as phrasing cues, and sequence elements. Since learning throughout the pattern depends on a common process, MK-801 should not differentially impair and spare learning for different elements within a pattern. Rather, the prediction would be that learning for all elements within the pattern should be affected or, alternatively, none should. In Experiment 1, MK-801 produced little effect on learning for elements within chunks, but it produced profound impairment for a violation elementthat is, a pattern element that violated pattern structure. Second, in Experiment 3, MK-801 disrupted the integration of new pattern information with old pattern information only when the new information was inconsistent with earlier pattern structure. This result is not easily explained by the discrimination learning view alone because no distinctive stimulus attributes identify added elements as structurally consistent "perfect" or structurally inconsistent "violation" elements; distinguishing between perfect and violation elements depends entirely on recognizing the formal relationship of the added element to rest of the pattern (cf. Fountain \& Rowan, 1995b). These two apparent dissociations of rule and discrimination learning observed in initial acquisition (Experiment 1) and in integrating new pattern information with old (Experiment 3 ) would seem to contradict the unitary process view that discrimination learning processes alone can account for rat serial pattern learning phenomena. Instead, they favor a dual-process view that posits both discrimination learning and rule learning mechanisms in serial pattern learning (Fountain, 1986; Fountain et al., 1985).

Other theories of hippocampal function also have an associative flavor not dissimilar to that of the discrimination learning view of Capaldi et al. (1984). Recent neurobehavioral and computational evidence has been presented in support of the hypothesis that the hippocampus mediates associations between discontiguous events (Rawlins, 1985; Wallenstein, Eichenbaum, \& Hasselmo, 1998). Wallenstein et al. (1998) suggest that associations between events that are either temporally discontiguous (greater than $100 \mathrm{msec}$ apart) or spatially discontiguous may depend on the formation of "context fields" that can bridge temporal or spatial discontiguities and that hippocampus and some cortical areas should have the capacity to develop context fields. One concern for the present paper is that the Wallenstein et al. computational model is based on hippocampal area CA3 cytoarchitectural details, and area CA3 LTP is not mediated by NMDA receptors (Harris \& Cotman, 1986). Thus, MK-801 should not affect CA3 plasticity or related processes. However, even if context fields can develop in areas where LTP is mediated by NMDA receptors, this notion does not provide an adequate description of the MK-801 induced impairments reported in Experiments 1-3. First, all pattern elements were separated by at least $1 \mathrm{sec}$, so a general impairment due to hippocampal dysfunction would be expected in ac- 
quisition, and this was not observed. Second, this hypothesis might suggest that impairments should be correlated with the degree of discontiguity, so that, under conditions of hippocampal dysfunction, longer intervals should be more difficult to bridge associatively than shorter intervals. In fact, in Experiments 1 and 3, acquisition of the violation element that followed a 1 -sec ITI was much more difficult than acquisition of chunk boundary elements that followed 3-sec ITIs, a pattern incompatible with this view. This is not to say that hippocampus does not serve the function of creating "associations across time" (Rawlins, 1985 ), only that this associative view alone cannot adequately account for the results reported in Experiments 1 and 3.

Taken together, the foregoing phenomena indicate the limitations of the view that discrimination learning is the sole mechanism mediating serial pattern learning. The results seem to fit better with the notion that serial pattern learning is mediated by both discrimination learning and rule-learning processes that are differentially impaired by MK-801, as suggested in earlier work (Fountain, 1986; Fountain et al., 1985). According to this view, MK-801 impaired discrimination learning processes important for learning events that depended on stimulus-response relations-chunk boundary elements and violation elements - but MK-801 had little effect on rule learning. Whether or not the stimulus-response relations that were not learned under MK- 801 could be characterized as necessarily "discontiguous" was not directly assessed in these experiments, but learning for some events cued only by discontiguous events (viz., within chunk elements) was spared under some circumstances-namely, when they could also be described as highly structured.

If rule learning is spared by MK- 801 , what processes are impaired by suppression of NMDA receptor mediated plasticity that can account for the discrimination learning deficits described in Experiments 1 and 3? Learning for simple spatial or nonspatial discrimination problems is rarely found to be impaired by hippocampal lesions or NMDA receptor antagonists (Alvarado \& Rudy, 1995c; Rawlins, Lyford, Seferiades, Deacon, \& Cassaday, 1993), yet we report discrimination learning deficits for chunk boundaries and the violation element of serial patterns. One possible explanation for our results is that anticipating chunk boundaries and the violation element required the kind of nonlinear "configural" discriminations that are often impaired by hippocampal lesions (Alvarado \& Rudy, 1995a, 1995b, 1995c; Rudy \& Sutherland, 1995). Nonlinear discriminations are "conditional" in the sense that they require conditional relations among multiple cues to guide responding. In other studies, rats learning the serial patterns used in the present experiments have shown that they depended heavily on phrasing cues to guide the response immediately following the cue, but cue removal also revealed that pattern elements also contributed to guiding the response (cf. Stempowski et al., 1999). Recent work in our lab indicates that when rats learn sequences comparable to those used by Stempowski et al., after train- ing with phrasing cues, they are sensitive to changes in chunk length when responding to phrasing cues (Fountain, Muller, \& Wallace, 1999), suggesting that a compound of the temporal phrasing cue and chunk length cues are responsible for guiding responses at cued chunk boundaries. Similarly, to anticipate the violation element, rats may have used multiple cues, such as serial position or timing, phrasing cues, and spatial or apparatus cues, to guide responses, because no one cue was sufficient to solve their "branching" sequential problem adequately. The basis of hippocampal-lesioned rats' difficulties with configural discriminations is still an open question (Alvarado \& Rudy, 1995b; Astur \& Sutherland, 1998; Rudy \& Sutherland, 1995). Nonetheless, it is worth noting that the parallels between configural discrimination learning deficits induced by hippocampal lesions and the deficits reported here for MK-801 are consistent with the idea that MK-801 impaired serial learning by suppressing plasticity in the hippocampus.

\section{Discrimination Learning, Rule Learning, and Hippocampal-Mediated "Representational Flexibility"}

Fountain (Fountain, 1986; Fountain et al., 1985) proposed a dual-process model that was developed specifically to describe the processes rats use in serial pattern learning. Fountain et al. (1985) proposed, as we have in this paper, that both rote learning (discrimination learning) and rule learning may play important roles in serial learning, depending on the nature of the pattern to be learned. Furthermore, Fountain et al. (1985) proposed that the hippocampal formation plays an important role in at least some forms of discrimination learning, but not in rule learning processes. This discrimination learning versus rule learning hypothesis has been used to explain a variety of behavioral serial pattern learning phenomena (Fountain, 1990; Fountain \& Rowan, 1995a, 1995b). It has also been used to explain why in earlier work learning for formally simple (highly structured) serial patterns was unimpaired, whereas learning for formally complex (unstructured) patterns was impaired following hippocampal damage (Fountain, 1986; Fountain et al., 1985).

Because MK- 801 was administered systemically in the experiments described above, the effects of the drug cannot be conclusively attributed to a disruption of function of any one brain structure nor to any simple pharmacological effect (i.e., affecting only NMDA receptors without interactions or effects on other receptor systems). Thus, in Experiments 1-3, MK-801 may have affected brain areas other than hippocampus and processes other than NMDA-mediated plasticity in hippocampus to influence the results obtained in the three experiments. However, the similarity between the learning deficits reported here and those produced by other manipulations that affect hippocampal function (Fountain et al., 1985) strongly suggests that the deficits observed in Experiments $1-3$ were produced in large measure by MK- 801 antagonism of the NMDA receptors found in the hip- 
pocampus and related memory systems. In particular, in Experiments 1 and 3, acquisition for structured elements of patterns (mediated by rule learning) was unimpaired by MK-801, whereas acquisition for elements that violated simple structure (mediated by discrimination learning) was impaired, a pattern of results observed by Fountain et al. (1985) in hippocampal-damaged rats.

Of particular interest is the result from Experiment 3 showing that new information disrupts performance on previously learned material if it is inconsistent with the structure of the previously learned pattern. One interpretation of these results is that adding new information to a pattern representation is possible under MK-801, but only if the information is consistent with pattern structure that has already been encoded. In fact, this initial evidence indicates that, for rats with MK-801 induced NMDA receptor blockade, new information that is structurally inconsistent can disrupt previously well learned response patterns. This suggests that, in intact rats, non-NMDA systems mediate rule induction but that these systems are not entirely independent of NMDA receptor mediated learning processes. Specifically, Experiment 3 showed that NMDA-mediated processes, perhaps involving hippocampus, may play a role in the successful integration of new rule-inconsistent serial pattern information with already-encoded information about pattern structure. These ideas are reminiscent of the distinction between flexible and inflexible memory processes proposed by Eichenbaum, Otto, and Cohen (1992), but our MK-801 results suggest that what constitutes "representational flexibility" is far from resolved. Under MK-801, rats were able to add a rule-consistent chunk to their already-learned pattern with relatively little difficulty, but not a rule-inconsistent chunk.

A closer look at the results of Experiments 1 and 3 suggests some novel notions of representational flexibility. In Experiment 1, MK-801 produced deficits in pattern acquisition for chunk boundaries and, most acutely, for an element that violated pattern structure. This impairment was observed despite the fact that neither the sequential pattern nor the spatial and temporal cues associated with the pattern ever changed from day to day. These conditions would seem to favor encoding of all pattern elements by memory systems variously termed reference memory (Olton et al., 1984), procedural memory (Cohen \& Squire, 1980), and, more recently, nondeclarative memory - the latter characterized as a memory system biased toward "inflexible" rather than "flexible" representations (Eichenbaum et al., 1992). Yet, under these conditions of highly repetitive presentation of sequences of events, MK-801 produced clear deficits in learning for some pattern elements but not others. Similarly, in the transfer phase of Experiment 3, a chunk added to a welllearned pattern under MK-801 had little impact on performance of the well-learned portion of the pattern, and performance was well above chance for the newly added information as long as the information was consistent with the structure of the well-learned pattern. In the trans- fer, the rats were required to make new responses at a time and place not previously trained, yet they showed no ill effects of this challenge to representational flexibility unless the required response violated pattern structure. These results do not argue against the notion of representational flexibility; they argue that the content of the memorial representation is, in this case, nonassociative, structural information describing serial patterns (cf. Fountain, 1990; Fountain \& Annau, 1984; Fountain \& Hulse, 1981; Fountain \& Rowan, 1995a, 1995b; Fountain et al., 1985; Hulse, 1978).

The rule learning versus discrimination learning dualprocess view is novel in the literature on animal models of memory function and dysfunction in that it predicts which learning/memory system will be used to store information on the basis of the structural characteristics of the information to be encoded. This model assumes that rats, like humans, are sensitive to structural relationships that describe patterns of stimuli and that processes for encoding such information are dissociable from discrimination learning processes, particularly those mediated by NMDA receptor dependent systems of plasticity. This dual-process view of serial learning should not be construed as attempting to supplant current notions of hippocampal involvement in representational flexibility (Eichenbaum et al., 1992) and association formation for discontiguous events (Rawlins, 1985; Wallenstein et al., 1998). Instead, the evidence from these experiments and others demonstrating similar dissociations of rule-based versus nonrulebased learning and memory (e.g., Cohen, 1984) should not be overlooked in formulating more general models seeking to characterize the fundamental nature of learning and memory systems mediated by different brain systems.

\section{REFERENCES}

Alvarado, M. C., \& Rudy, J. W. (1995a). A comparison of "configural" discrimination problems: Implications for understanding the role of the hippocampal formation in learning and memory. Psychobiology, 23, 178-184

Alvarado, M. C., \& Rudy, J. W. (1995b). A comparison of kainic acid plus colchicine and ibotenic acid-induced hippocampal formation damage on four configural tasks in rats. Behavioral Neuroscience, 109, 1052-1062.

Alvarado, M. C., \& Rudy, J. W. (1995c). Rats with damage to the hippocampal-formation are impaired on the transverse-patterning problem but not on elemental discriminations. Behavioral Neuroscience, 109, 204-211.

Astur, R. S., \& SutherLand, R. J. (1998). Configural learning in humans: The transverse patterning problem. Psychobiology, 26, 176-182.

CAPALDI, E. J. (1985). Anticipation and remote associations: A configural approach. Journal of Experimental Psychology: Learning, Memory, \& Cognition, 11, 444-449.

CaPALDI, E. J. (1994). The sequential view: From rapidly fading stimulus traces to the organization of memory and the abstract concept of number. Psychonomic Bulletin \& Review, 1, 156-181.

Capaldi, E. J., \& Molina, P. (1979). Element discriminability as a determinant of serial-pattern learning. Animal Learning \& Behavior, 7 , 318-322.

Capaldi, E. J., Verry, D. R.. NaWrocki, T. M.. \& Miller, D. J. (1984). Serial learning, interitem associations, phrasing cues, interference, overshadowing, chunking, memory, and extinction. Animal Learning \& Behavior. 12, 7-20. 
Caramanos, Z., \& Shapiro, M. L. (1994). Spatial memory and $N$ methyl-D-aspartate receptor antagonists APV and MK-801: Memory impairments depend on familiarity with the environment, drug dose, and training duration. Behavioral Neuroscience, 108, 30-43.

Carlezon, W. A., \& WISE, R. A. (1993). Morphine-induced potentiation of brain stimulation reward is enhanced by MK-801. Brain Research, 620, 339-342.

COHEN, N. J. (1984). Preserved learning capacity in amnesia: Evidence for multiple memory systems. In N. Butters \& L. R. Squire (Eds.), The neuropsychology of memory (pp. 83-103). New York: Guilford.

CoHen, N. J., \& SQuiRe, L. R. (1980). Preserved learning and retention of pattern-analyzing skill in amnesia: Dissociation of knowing how and knowing that. Science, 210, 207-210.

Compton, D. M. (1993). Encoding of a nonmonotonic serial pattern: Role of the dorsal hippocampus and amygdala. Physiology \& Behavior, 53, 657-665.

Eichenbaum, H., Otto, T., \& Cohen, N. J. (1992). The hippocampusWhat does it do? Behavioral \& Neural Biology, 57, 2-36.

Fountain, S. B. (1986). Serial-pattern learning: A unitary process? Physiological Psychology, 14, 67-70.

Fountain, S. B. (1990). Rule abstraction, item memory, and chunking in rat serial-pattern tracking. Journal of Experimental Psychology: Animal Behavior Processes, 16, 96-105.

Fountain, S. B., \& ANnaU, Z. (1984). Chunking, sorting, and rulelearning from serial patterns of brain-stimulation reward by rats. $A n$ imal Learning \& Behavior, 12, 265-274.

Fountain, S. B., Evensen, J. C., \& HulSE, S. H. (1983). Formal structure and pattern length in serial pattern learning by rats. Animal Learning \& Behavior, 11, 186-192.

Fountain, S. B., Henne, D. R., \& Hulse, S. H. (1984). Phrasing cues and hierarchical organization in serial pattern learning by rats. Journal of Experimental Psychology: Animal Behavior Processes, 10, 3045.

Fountain, S. B., \& Hulse, S. H. (1981). Extrapolation of serial stimulus patterns by rats. Animal Learning \& Behavior, 9, 381-384.

Fountain, S. B., Krauchunas, S. M., \& Rowan, J. D. (1999). Serialpattern learning in mice: Pattern structure and phrasing. Psycholog ical Record, 49, 173-192.

Fountain, S. B., Muller, M. S., \& Wallace, D. G. (1999). Determinants of pattern tracking in rats. Manuscript in preparation.

Fountain, S. B., \& Rowan, J. D. (1995a). Coding of hierarchical versus linear pattern structure in rats and humans. Journal of Experimental Psychology: Animal Behavior Processes, 21, 187-202.

Fountain, S. B., \& Rowan, J. D. (1995b). Sensitivity to violations of "run" and "trill" structures in rat serial-pattern learning. Journal of Experimental Psychology: Animal Behavior Processes, 21, 78-81.

Fountain, S. B., Schenk, D. E., \& AnnaU, Z. (1985). Serial-patternlearning processes dissociated by trimethyltin exposure in rats. Physiological Psychology, 13, 53-62.

HAGGBLOOM, S. J. (1985). Serial learning and transfer in rats: Effects of changes in stimulus-stimulus associations, pattern structure, and serial position information. Animal Learning \& Behavior, 13, 370-374.

HAGgBloom, S. J., \& Brooks, D. M. (1985). Serial anticipation and pattern extrapolation in rats as a function of element discriminability. $A n$ imal Learning \& Behavior, 13, 303-308.

Hargreaves, E. L., Cote, D., \& Shapiro, M. L. (1997). A dose of MK801 previously shown to impair spatial learning in the radial maze attenuates primed burst potentiation in the dentate gyrus of freely moving rats. Behavioral Neuroscience, 111, 35-48.

HARRIS, E. W., \& CotMAN, C. W. (1986). Long-term potentiation of guinea pig mossy fiber responses is not blocked by $N$-methyl-D-aspartate antagonists. Neuroscience Letters, 70, 132-137.

HeRberG, L. J., \& Rose, I. C. (1989). The effect of MK-801 and other antagonists of NMDA-type glutamate receptors on brain-stimulation reward. Psychopharmacology, 99, 87-90.

Hulse, S. H. (1978). Cognitive structure and serial pattern learning by animals. In S. H. Hulse, H. Fowler, \& W. K. Honig (Eds.), Cognitive processes in animal behavior (pp. 311-340). Hillsdale, NJ: Erlbaum.
HULSE, S. H., \& DoRSKY, N. P. (1977). Structural complexity as a determinant of serial pattern learning. Learning \& Motivation, 8, 488-506.

HuLSE, S. H., \& DORSKY, N. P. (1979). Serial pattern learning by rats: Transfer of a formally defined stimulus relationship and the significance of nonreinforcement. Animal Learning \& Behavior, 7, $211-220$.

KESNER, R. P., \& NovaK, J. M. (1982). Serial position curve in rats: Role of the dorsal hippocampus. Science, 218, 173-175.

KIRK, R. E. (1968). Experimental design: Procedures for the behavioral sciences. Belmont, CA: Brooks/Cole.

KNOPMAN, D. S., \& NisSEN, M. J. (1987). Implicit learning in patients with probable Alzheimer's disease. Neurology, 37, 784-788.

LEE, H., \& KIM, J. J. (1998). Amygdalar NMDA receptors are critical for new fear learning in previously fear-conditioned rats. Journal of Neuroscience, 18, 8444-8454.

OLDS, M. E. (1996). Dopaminergic basis for the facilitation of brain stimulation reward by the NMDA receptor antagonist, MK-801. European Journal of Pharmacology, 306, 23-32.

Olton, D. S., Shapiro, M. L., \& Hulse, S. H. (1984). Working memory and serial patterns. In H. L. Roitblat, T. G. Bever, \& H. S. Terrace (Eds.), Animal cognition (pp. 171-182). Hillsdale, NJ: Erlbaum.

Phelps, M. T., \& RoberTs, W. A. (1991). Pattern tracking on the radial maze: Tracking multiple patterns at different spatial locations. Journal of Experimental Psychology: Animal Behavior Processes, 17, 411-422.

Rawlins, J. N. P. (1985). Associations across time: The hippocampus as a temporary memory store. Behavioral \& Brain Sciences, 8, 479496.

Rawlins, J. N. P., Lyford, G. L., Seferiades, A., Deacon, R. M. J., \& CASSADAY, H. J. (1993). Critical determinants of nonspatial working memory deficits in rats with conventional lesions of the hippocampus or fornix. Behavioral Neuroscience, 107, 420-433.

Rowan, J. D., Toner, B. S., \& Fountain, S. B. (1992). MK-801 enhances brain-stimulation reward and impairs serial pattern learning in rats. Toxicologist, 12, 273.

RudY, J. W., \& SutherLand, R. J. (1995). Configural association theory and the hippocampal formation: An appraisal and reconfiguration. Hippocampus, 5, 375-389.

Shapiro, M. L., \& Caramanos, Z. (1990). NMDA antagonist MK-801 impairs acquisition but not performance of spatial working and reference memory. Psychobiology, 18, 231-243.

ShaPIRO, M. L., \& O'ConNor, C. (1992). $N$-methyl-D-aspartate receptor antagonist MK-801 and spatial memory representation: Working memory is impaired in an unfamiliar but not in a familiar environment. Behavioral Neuroscience, 106, 604-612.

Stempowski, N. K., Carman, H. M., \& Fountain, S. B. (1999). Temporal phrasing and overshadowing in rat serial-pattern learning. Learning \& Motivation, 30, 74-100.

Stevens, L., Shapiro, M. [L.], \& White, N. M. (1997). Effects of NMDA receptor blockade on behaviors differentially affected by fimbria/fornix and amygdala lesions. Psychobiology, 25, 109-117.

TERRACE, H. S. (1987). Chunking by a pigeon in a serial learning task. Nature, 325, 149-151.

TERRACE, H. S. (1991). Chunking during serial learning by a pigeon: I. Basic evidence. Journal of Experimental Psychology: Animal Behavior Processes, 17, 81-93.

TEYler, T. J., \& DiScenNa, P. (1987). Long-term potentiation. Annual Review of Neuroscience, 10, 131-161.

Wallenstein, G. V., Eichenbaum, H., \& Hasselmo, M. E. (1998). The hippocampus as an associator of discontiguous events. Trends in Neurosciences, 21, 317-323.

White, A. M., \& BEST, P. J. (1998). The effects of MK-801 on spatial working memory and within-session spatial learning. Pharmacology. Biochemistry \& Behavior, 59, 613-617.

(Manuscript received November 19, 1998; revision accepted for publication July 21, 1999.) 